\title{
Quantum dynamics of a molecular matter-wave amplifier
}

\author{
Jing Cheng ${ }^{1}$ and YiJing Yan $^{2}$ \\ ${ }^{1}$ School of Physical Science and Technology, South China University of Technology, Guangzhou 510640, China \\ ${ }^{2}$ Department of Chemistry, Hong Kong University of Science and Technology, Kowloon, Hong Kong
}

(Received 6 November 2006; published 26 March 2007)

\begin{abstract}
We study the quantum dynamics of a model of molecular matter-wave amplifier proposed by Search and Meystre [Phys. Rev. Lett. 93, 140405 (2004)], which employs a strongly damped optical cavity to convert an atomic Bose-Einstein condensate into vibrational ground-state molecules. By using the Monte Carlo wavefunction method, we calculate the quantum evolution of the atomic and molecular matter waves. We find that the system always evolves into a pure ground-state molecular matter wave even if initially there was no ground-state molecules. Statistical properties of these atomic and molecular matter waves are also investigated. The final state of the molecular field is sub-Poissonian. Two-mode correlation functions exhibit anticorrelated properties.
\end{abstract}

DOI: 10.1103/PhysRevA.75.033614

PACS number(s): 03.75.-b, 42.50.Lc, 05.30.Jp

\section{INTRODUCTION}

Coherent generation of ultracold molecules or molecular Bose-Einstein condensates (BECs) has been an active research field in matter-wave and cold-atomic physics [1-3]. Both condensed bosonic atoms and degenerate fermionic atoms can be converted into molecules by using either the Feshbach resonance (FR) method or photoassociation (PA) process. Studies on cold molecules or molecular BECs may lead to new scientific developments such as the quantum superchemistry [4,5], BEC-BCS crossover [6], and matterwave entanglement [7]. Experimental observations of molecular BECs have been realized in several groups [2]. Theoretically, the mean-field Gross-Pitaevski equations (GPEs) have been widely used to describe the dynamical evolution of coupled atom-molecule BECs [8-11]. Moreover, atommolecule BECs may also show quantum wave statistics $[5,12-16]$. Thus, the study on quantum dynamics and statistical correlations of atom and molecule BECs is of great importance in the development of quantum optics of molecules [17].

Generally, the lifetime of molecules formed from bosons via FR or PA are very short because they are lying on highly excited vibrational states [18]. It is crucial for practical applications to create molecules in their vibrational ground state. Several methods, including stimulated two-photon Raman PA [19], Bose-stimulated Raman adiabatic passage [20-23], and Feshbach stimulated Raman photoproduction [24], have been proposed to create ground-state molecule BEC.

Recently, Search and Meystre proposed a molecular matter-wave amplifier (MMWA) scheme, by using FR and two-photon Raman transition inside a poor quality cavity [25]. In their model, a bosonic atom pair are converted into a quasibound molecule via FR, then followed by a two-photon transition to transfer the quasibound molecules to groundstate molecules. Molecules in the quasibound state are first coupled to an excited molecular state by an intense optical field, then decay to the ground state with emission of a photon into a quantized cavity mode. High efficiency and unidirectional amplification of an initial ground-state molecular matter wave have been demonstrated via numerically solving the mean-field equations.

In this paper, we wish to know the quantum statistical properties of the MMWA. Based on the Lindblad's master equation description of the Search-Meystre model, we use the Monte Carlo wave-function method to study the quantum evolution of the system and calculate the second-order correlation functions of atomic and molecular fields. We show that even if there is no initial ground-state molecular fields, all atoms can still be converted completely into the groundstate molecules. We also show that the statistics of produced ground-state molecules is sub-Poissonian and the correlations between any two matter-wave fields are anticorrelated. We describe the MMWA model and numerical method in Sec. II, and present the main results and discussions in Sec. III. Conclusions are given in Sec. IV.

\section{FORMALISM}

The Search-Meystre's MMWA is modeled in terms of four matter-wave modes and one optical field mode [25]. Initially, all atoms are inside an optical cavity and Bose condensed into the state $|0\rangle$. Two atoms in $|0\rangle$ can be converted to a molecule in the quasibound state $|1\rangle$ via FR. Then a classical laser couples state $|1\rangle$ to an electronically excited molecular state $|2\rangle$. Transition from $|2\rangle$ to the molecular ground state $|3\rangle$ is realized by a quantized mode of the cavity field. In the original model of Feshbach stimulated photoproduction [24], the $|2\rangle \rightarrow|3\rangle$ transition is also coupled by a classical optical field. The use of a quantized cavity mode in this MMWA model can suppress other unwanted transitions. Under the assumptions that the cavity mode is strongly damped and there are large frequency detunings in transitions $|1\rangle \rightarrow|2\rangle$ and $|2\rangle \rightarrow|3\rangle$, the dynamical evolution of a MMWA can be described by the master equation given in Ref. [25] as

$$
\begin{aligned}
\dot{\rho}= & -i\left[H_{01}+\delta \hat{b}_{3}^{\dagger} \hat{b}_{3}, \rho\right]+\kappa|\beta|^{2}\left(\hat{b}_{1} \hat{b}_{3}^{\dagger} \rho \hat{b}_{3} \hat{b}_{1}^{\dagger}-\hat{b}_{3} \hat{b}_{1}^{\dagger} \hat{b}_{1} \hat{b}_{3}^{\dagger} \rho\right. \\
& + \text { H. . . }) .
\end{aligned}
$$

Here, $\hat{b}_{0}, \hat{b}_{1}$, and $\hat{b}_{3}$ are bosonic annihilation operators for 
atoms or molecules in the states $|0\rangle,|1\rangle$, and $|3\rangle$, respectively. The FR coupling is described by $H_{01}=\alpha \hat{b}_{1}^{\dagger} \hat{b}_{0}^{2} e^{i \Delta t}+$ H.c., with $\alpha$ and $\Delta$ being the coupling strength and FR detuning. In Eq. (1), $\kappa$ denotes the cavity mode damping rate, while $\delta$ and $\beta \ll 1$ are the two-photon detuning and relative coupling strength for the $|1\rangle \rightarrow|3\rangle$ transition, respectively. The intermediate off-resonant-state $|2\rangle$ is dark and does not actively participate in Eq. (1).

The complete information about the dynamics of the MMWA can be obtained by solving Eq. (1). But this is very difficult. Instead, in Ref. [25], truncated equations of motion for operator expectation values were considered. It has been shown that an unidirectional amplification of a small initial population in $|3\rangle$ can be realized. Apparently, this mean-field method cannot tell us the quantum statistics of the amplified ground-state molecular field.

In this work, we exploit the theory of quantum trajectories [26], and study the quantum dynamics of MMWA by using the Monte Carlo wave-function method. This is done by recognizing that Eq. (1) has the Lindblad form

$$
\dot{\rho}=-i\left[H_{01}+\delta \hat{b}_{3}^{\dagger} \hat{b}_{3}, \rho\right]+L_{\text {relax }}(\rho),
$$

with the Lindblad dissipation being associated with the quantum jump operator, $\hat{C}=\sqrt{2 \kappa|\beta|^{2}} \hat{b}_{1} \hat{b}_{3}^{\dagger}$; i.e. [26],

$$
L_{\text {relax }}(\rho)=-\frac{1}{2}\left(\hat{C}^{\dagger} \hat{C} \rho+\rho \hat{C}^{\dagger} \hat{C}-2 \hat{C}^{\dagger} \rho \hat{C}\right) .
$$

As a result, the initial pure state in each trajectory evolves with the non-Hermitian $H=\left(H_{01}+\delta \hat{b}_{3}^{\dagger} \hat{b}_{3}\right)-i \hat{C}^{\dagger} \hat{C} / 2$, together with a series of random quantum jumps [26]. Apparently, the state remains a pure state in each trajectory, and the expectation value of any operator can be calculated from the ensemble average of quantum trajectories. Note that the quantum jump operator $\hat{C}$ conserves the total population, i.e., $M$ $=N_{0} / 2+N_{1}+N_{3}$ remains a constant; $N_{2}=0$ since the offresonant-state $|2\rangle$ does not participate in Eq. (1) explicitly.

The stochastic wave function can now be expressed as

$$
|\phi(t)\rangle=\sum_{n=0}^{M} \sum_{m=0}^{M-n} c_{n m}(t)|2 n\rangle_{0}|M-n-m\rangle_{1}|m\rangle_{3} .
$$

Here, $|N\rangle_{j}$ denotes the basis wave function for the specified bosonic state $|j\rangle$ being occupied by $N$ particles. According to the standard algorithm [26,27], the jump probability is given by $\delta p=\delta t\left\langle\phi(t)\left|\hat{C}^{\dagger} \hat{C}\right| \phi(t)\right\rangle$ and the stochastic propagator for $|\phi(t+\delta t)\rangle=U(t+\delta t, t)|\phi(t)\rangle$ is

$$
U(t+\delta t, t)= \begin{cases}\frac{1-i H \delta t}{\sqrt{1-\delta p}}, & \text { with probability } 1-\delta p, \\ \frac{\hat{C}}{\sqrt{\delta p / \delta t}}, & \text { with probability } \delta p .\end{cases}
$$

The mean occupation numbers in the individual states can now be evaluated as

$$
\begin{gathered}
n_{0}(t)=\operatorname{Tr}\left[\hat{b}_{0}^{\dagger} \hat{b}_{0} \rho\right]=\sum_{n=0}^{M} \sum_{m=0}^{M-n} 2 n \overline{\left|c_{n m}(t)\right|^{2}}, \\
n_{1}(t)=\operatorname{Tr}\left[\hat{b}_{1}^{\dagger} \hat{b}_{1} \rho\right]=\sum_{n=0}^{M} \sum_{m=0}^{M-n}(M-n-m) \overline{\left|c_{n m}(t)\right|^{2}}, \\
n_{3}(t)=\operatorname{Tr}\left[\hat{b}_{3}^{\dagger} \hat{b}_{3} \rho\right]=\sum_{n=0}^{M} \sum_{m=0}^{M-n} m \overline{\left|c_{n m}(t)\right|^{2}} .
\end{gathered}
$$

Here, $\overline{\left|c_{n m}(t)\right|^{2}}$ denotes the trajectory ensemble average of the stochastic wave-function amplitude. We can also calculate the equal-time second-order correlation functions,

$$
\begin{gathered}
G_{j}^{(2)}(t)=\operatorname{Tr}\left[\hat{b}_{j}^{\dagger} \hat{b}_{j}^{\dagger} \hat{b}_{j} \hat{b}_{j} \rho\right], \quad j=0,1,3, \\
G_{k j}^{(2)}(t)=\operatorname{Tr}\left[\hat{b}_{k}^{\dagger} \hat{b}_{j}^{\dagger} \hat{b}_{j} \hat{b}_{k} \rho\right], \quad k \neq j,
\end{gathered}
$$

for the single-mode matter wave in the specified state $|j\rangle$, or the two-mode matter wave in different $|k\rangle$ and $|j\rangle$. These correlation functions are widely used in quantum optics to characterize the quantum statistics of optical fields [28]. Generally speaking, the field is sub-Poisonnian if $G_{j}^{(2)}<n_{j}^{2}$, the signature of a nonclassical matter-wave field. Moreover, the violation of the Cauchy-Schwarz inequality, $G_{k j}^{(2)}$ $>\sqrt{G_{k}^{(2)} G_{j}^{(2)}}$, implies that the two matter-wave fields are nonclassically correlated [28].

\section{NUMERICAL RESULTS AND DISCUSSIONS}

Presented in this section are the simulated results of MMWA, exemplified by the system with the resonant detunings $(\Delta=\delta=0)$ and the relative FR coupling strength of $\alpha$ $=10 \kappa|\beta|^{2}$. The unit of time $t$ is set to be $1 /\left(\kappa|\beta|^{2}\right)$.

Let us start with the initial state, $|\phi(0)\rangle=|200\rangle_{0}|0\rangle_{1}|10\rangle_{3}$, comparable with that used in Ref. [25], in which the molecular ground state $|3\rangle$ has a small population initially. Note that the total population is $M=N_{0} / 2+N_{1}+N_{3}=110$.

Shown in Fig. 1(a) is a single trajectory, in terms of the quantum jump dynamics of the populations in the three matter-field modes. It is evident that $n_{3}(t)$ monotonically increases with $t$. As the non-Hermitian Hamiltonian $H$ commutes with $\hat{b}_{3}^{\dagger} \hat{b}_{3}$, the population in $|3\rangle$ in each trajectory remains a constant step value, until a jump that occurs randomly and brings one more molecule to $|3\rangle$. The trajectory of $|\phi(t)\rangle$ is not deterministic in general. However, in the present model of study the quantum jump is unidirectional and irreversible. The MMWA in each trajectory will sooner or later evolve into the pure ground-state molecular matter wave of $\left|\phi_{f}\right\rangle=|0\rangle_{0}|0\rangle_{1}|110\rangle_{3}$. In the trajectory depicted in Fig. 1(a) for example, the quantum state reaches at $\left|\phi_{f}\right\rangle$ around $t \approx 0.075$ [in the unit of $1 /\left(\kappa|\beta|^{2}\right)$ ], and remains there since then. Figure 1(b) presents the ensemble-averaged results. The resulting curves are similar to those in Ref. [25]. The evolutions of the average populations are smooth and the amplification of the matter wave field in $|3\rangle$ is monotonic towards the pure state of $\rho=\left|\phi_{f}\right\rangle\left\langle\phi_{f}\right|$. 

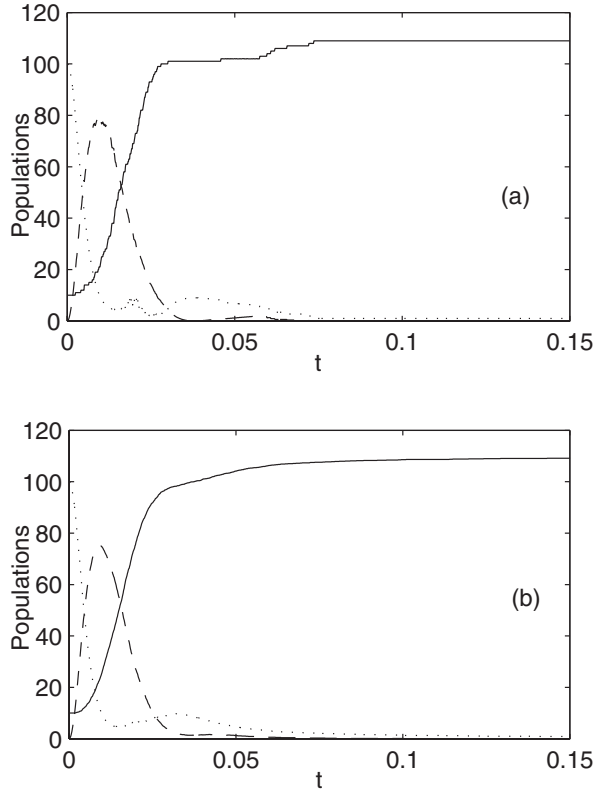

FIG. 1. Quantum evolution of the populations in the three states: $n_{0}(t) / 2$ (dotted curves), $n_{1}(t)$ (dashed curves), $n_{3}(t)$ (solid curves). (a) Results obtained from a single trajectory. (b) Results obtained by averaging over 50 trajectories.

In Fig. 2, we show the quantum fluctuations in the MMWA. Number statistics of the atoms and molecules are plotted in Fig. 2(a) in terms of the Mandel's $Q$ parameter,

$$
Q_{j}(t)=\frac{G_{j}^{(2)}(t)-n_{j}^{2}(t)}{n_{j}(t)},
$$

which is widely used in quantum optics. It is evident that the ground-state molecules will be of a sub-Poissonian distribution after a certain time. This is consistent with the previous discussion that the final state is a pure ground-state molecular number state. The atoms and quasibound molecules are initially in the sub-Poissonian and gradually transformed into super-Poissonian distributions until finally they are depleted. The joint quantum statistical properties of the three matterwave fields are reported in Fig. 2(b) in terms of $\Delta G_{k j}^{(2)}$ $=G_{k j}^{(2)}-\sqrt{G_{k}^{(2)} G_{j}^{(2)}}$. Curves at the early $(t<0.01)$ and later $(t$ $>0.05)$ time are trivial because of $G_{i j}^{(2)}(t) \approx 0$ and $G_{i}^{(2)}$ $\times(t) G_{j}^{(2)}(t) \approx 0$ there. It is also noticed that the two-mode correlation functions always satisfy the Cauchy-Schwarz inequality, $\Delta G_{i j}^{(2)}(t) \leq 0$. In other words, the matter-wave fields are completely anticorrelated. They do not tend to be created simultaneously. This property can be understood from the quantum trajectory analysis or the model itself that invokes the rotating-wave ansatz. The event of a quasibound molecule creation in $|1\rangle$ is closely related to that of the two atoms annihilation in $|0\rangle$. Thus, the populations in $|0\rangle$ and $|1\rangle$ cannot increase simultaneously. Similarly, the creation of a ground-state molecule in $|3\rangle$ requires a quasibound molecule in $|2\rangle$ be annihilated, and moreover, it is a competing event against the recovery of two atoms back to $|0\rangle$. Therefore, the matter wave in $|3\rangle$ is also antibouncing with respect to that in $|1\rangle$ and $|0\rangle$. Apparently, the fields in $|0\rangle$ and $|3\rangle$ are not di-
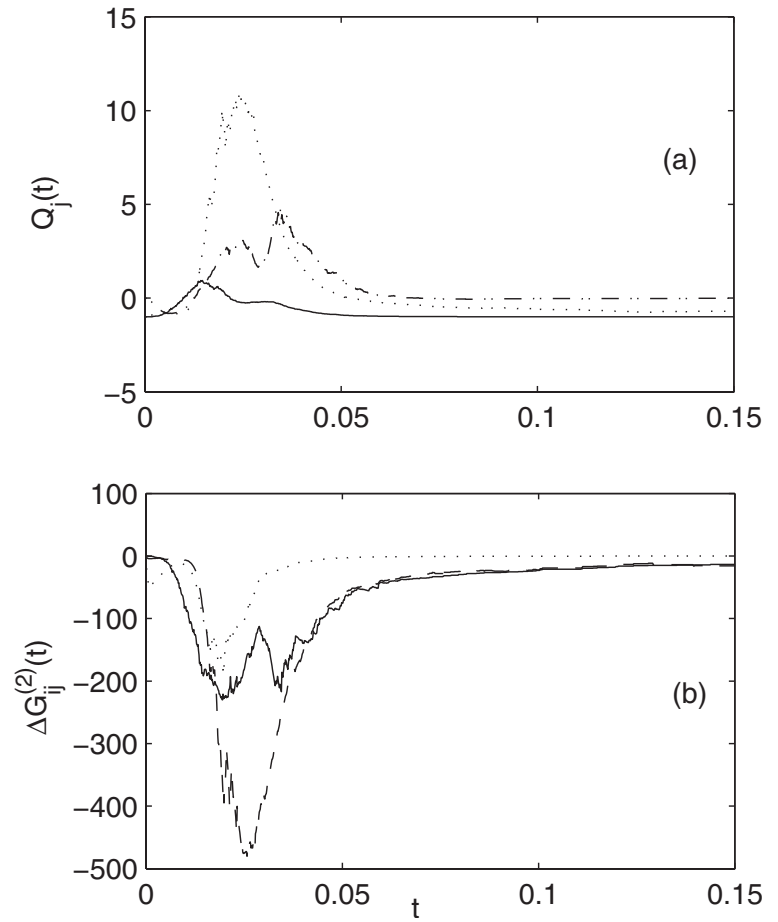

FIG. 2. (a) Mandel's $Q$ parameter as the function of time: $Q_{0}(t)$ (dotted curve), $Q_{1}(t)$ (dashed curve), $Q_{3}(t)$ (solid curve). (b) Quantum evolution of the two-mode correlation functions: $\Delta G_{01}^{(2)}(t)$ (dotted curve), $\Delta G_{03}^{(2)}(t)$ (dashed curve), $\Delta G_{13}^{(2)}(t)$ (solid line). All results are obtained by averaging over 50 trajectories.

rectly connected and their correlation is relatively small, as depicted in Fig. 2(b).

The observed MMWA properties above will remain all valid, even for the case where there were no initial population in the molecular ground state $|3\rangle$. Shown in Fig. 3 and Fig. 4 are the counterparts of Fig. 1 and Fig. 2, respectively,
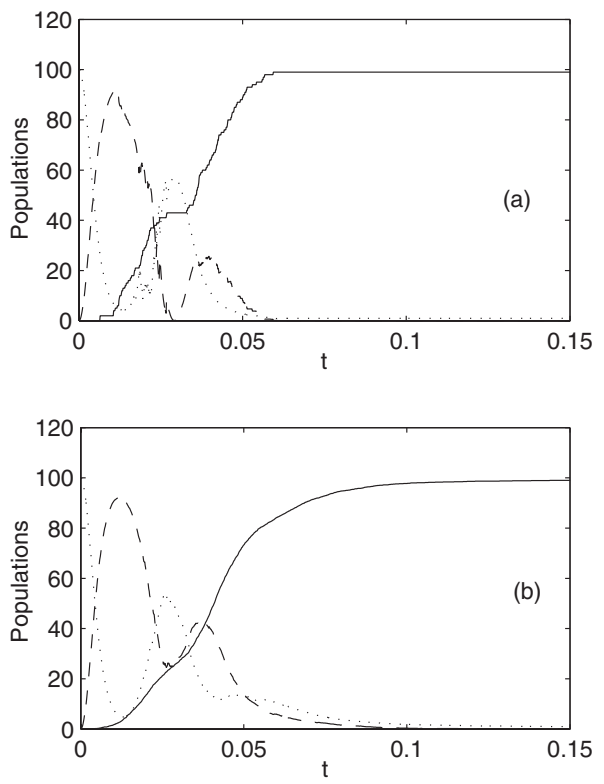

FIG. 3. The same as Fig. 1, but with the initial state of $\phi(t$ $=0)=|200\rangle_{0}|0\rangle_{1}|0\rangle_{3}$. 

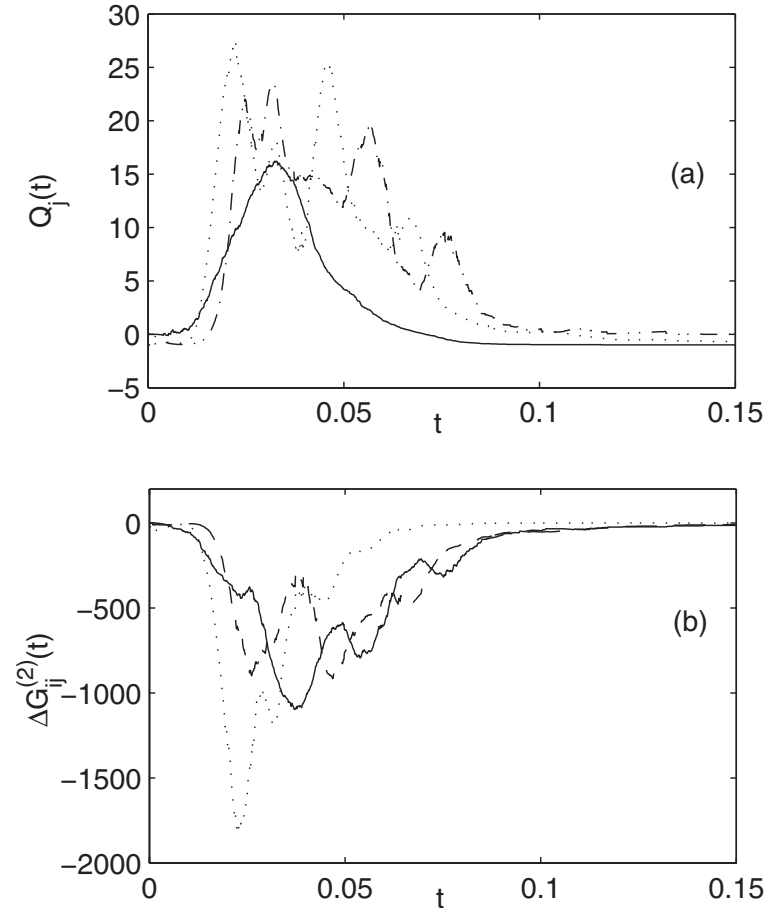

FIG. 4. The same as Fig. 2, but with the initial state of $\phi(t$ $=0)=|200\rangle_{0}|0\rangle_{1}|0\rangle_{3}$.

with the initial state being instead $|\phi(0)\rangle=|200\rangle_{0}|0\rangle_{1}|0\rangle_{3}$. These figures have similar qualitative behaviors. Note that the total population is now $M=N_{0} / 2+N_{1}+N_{3}=100$, rather than 110. All other parameters are not changed. As the quantum jumps into $|3\rangle$ occur spontaneously, the conversion of atoms to ground state molecules can always be realized in the present model of study, regardless whether there is an initial population or not in the molecular ground state. An initial seed in $|3\rangle$ just speeds up the conversion process and reduces the fluctuation.

\section{CONCLUSION AND DISCUSSION}

We have studied the quantum dynamics of a molecular matter-wave amplifier using the Monte Carlo wave-function method. We find that the amplification or generation of the ground-state molecular matter wave can be realized via random quantum jumps or the spontaneous emission in the present case. The conversion of near-unit efficiency can be achieved no matter if there is an initial population in the molecular ground state or not. Statistical properties of the matter waves are also investigated by calculating the equal time second-order correlation functions. The atomic and molecular matter waves can be super-Poissonian, and the desired final ground-state molecular field always show subPoissonian statistics. Any two-mode correlation functions satisfy the Cauchy-Schwarz inequality, indicating that any two matter fields are anticorrelated according to their joint quantum statistics.

The ground-state molecular field is sub-Possonian means it is antibunching in a certain time interval. But numerically determining this time scale requires one to know the two time correlation functions, which is very tedious and beyond the scope of the current paper, so we left it for the future work. Very recently, Nunnenkamp, Meiser, and Meystre [29] have studied the models of the ground-state heteronuclear molecules generated from two BECs, a mixture of a BEC and a degenerate fermion, and two degenerate fermions. Population evolutions are obtained via directly solving the master equations. But the second-order moments are calculated only in the short-time limit. So the quantum statistics of the molecules in the long-time evolution are not studied. On the other hand, in our paper, we use the Monte Carlo wavefunction method to calculate the long-time behaviors of the second order moments and the cross correlations between different atomic and molecular modes. We hope this method will be applied also to the models considered in Ref. [29].

\section{ACKNOWLEDGMENTS}

Support from the National Natural Science Foundation of China (Grant No. 10404031), South China University of Technology, and the Research Grants Council of Hong Kong Government (Grant No. 604804) is acknowledged.
[1] R. Wynar et al., Science 287, 1016 (2000); E. A. Donley, N. R. Claussen, S. T. Thompson, and C. E. Wieman, Nature (London) 417, 529 (2002); S. Durr, T. Volz, A. Marte, and G. Rempe, Phys. Rev. Lett. 92, 020406 (2004).

[2] M. Greiner, C. A. Regal, and D. S. Jin, Nature (London) 426, 537 (2003); M. W. Zwierlein, C. A. Stan, C. H. Schunck, S. M. F. Raupach, S. Gupta, Z. Hadzibabic, and W. Ketterle, Phys. Rev. Lett. 91, 250401 (2003); S. Jochim et al., Science 302, 2101 (2003).

[3] R. A. Duine and H. T. C. Stoof, Phys. Rep. 396, 115 (2004); E. Timmermans, P. Tommasini, M. Hussein, and A. Kerman, ibid. 315, 199 (1999).

[4] D. J. Heinzen, R. Wynar, P. D. Drummond, and K. V. Kheruntsyan, Phys. Rev. Lett. 84, 5029 (2000).
[5] J. J. Hope and M. K. Olsen, Phys. Rev. Lett. 86, 3220 (2001).

[6] C. A. Regal, M. Greiner, and D. S. Jin, Phys. Rev. Lett. 92, 040403 (2004); M. Bartenstein, A. Altmeyer, S. Riedl, S. Jochim, C. Chin, J. H. Denschlag, and R. Grimm, ibid. 92, 120401 (2004); M. W. Zwierlein, C. A. Stan, C. H. Schunck, S. M. F. Raupach, A. J. Kerman, and W. Ketterle, ibid. 92, 120403 (2004).

[7] J. F. Corney and P. D. Drummond, Phys. Rev. Lett. 93, 260401 (2004).

[8] P. D. Drummond, K. V. Kheruntsyan, and H. He, Phys. Rev. Lett. 81, 3055 (1998).

[9] J. Javanainen and M. Mackie, Phys. Rev. A 59, R3186 (1999).

[10] A. Vardi, V. A. Yurovsky, and J. R. Anglin, Phys. Rev. A 64, 063611 (2001). 
[11] M. Holland, J. Park, and R. Walser, Phys. Rev. Lett. 86, 1915 (2001).

[12] A. Vardi, V. A. Yurovsky, and J. R. Anglin, Phys. Rev. A 64, 063611 (2001).

[13] A. P. Hines, R. H. McKenzie, and G. J. Milburn, Phys. Rev. A 67, 013609 (2003).

[14] G. R. Jin, C. K. Kim, and K. Nahm, Phys. Rev. A 72, 045602 (2005).

[15] D. Meiser and P. Meystre, Phys. Rev. Lett. 94, 093001 (2005).

[16] K. V. Kheruntsyan, Phys. Rev. Lett. 96, 110401 (2006).

[17] C. P. Search and P. Meystre, Prog. Opt. 47, 139 (2005).

[18] V. A. Yurovsky, A. Ben-Reuven, P. S. Julienne, and C. J. Williams, Phys. Rev. A 60, R765 (1999).

[19] P. S. Julienne, K. Burnett, Y. B. Band, and W. C. Stwalley, Phys. Rev. A 58, R797 (1998).

[20] M. Mackie, R. Kowalski, and J. Javanainen, Phys. Rev. Lett. 84, 3803 (2000).
[21] P. D. Drummond, K. V. Kheruntsyan, D. J. Heinzen, and R. H. Wynar, Phys. Rev. A 65, 063619 (2002).

[22] H. Y. Ling, H. Pu, and B. Seaman, Phys. Rev. Lett. 93, 250403 (2004).

[23] J. Cheng, S. S. Han, and Y. J. Yan, Phys. Rev. A 73, 035601 (2006).

[24] M. Mackie, Phys. Rev. A 66, 043613 (2002).

[25] C. P. Search and P. Meystre, Phys. Rev. Lett. 93, 140405 (2004).

[26] M. Orszag, Quantum Optics (Springer-Verlag, Berlin, 2000), Chap. 16.

[27] M. B. Plenio and P. L. Knight, Rev. Mod. Phys. 70, 101 (1998), Sec. IV. C.

[28] D. F. Walls and G. J. Milburn, Quantum Optics (SpringerVerlag, Berlin, 1994).

[29] A. Nunnenkamp, D. Meiser, and P. Meystre, New J. Phys. 8, 88 (2006). 